\title{
Dietary patterns as predictors of childhood adiposity
}

\author{
C. A. Shaw ${ }^{1}$, J. Lara ${ }^{1}$, A. J. Adamson ${ }^{2}$, A. R. Jones ${ }^{2}$ and J. C. Mathers ${ }^{1}$ \\ ${ }^{1}$ Human Nutrition Research Centre, Institute for Ageing and Health, Newcastle University, Newcastle upon Tyne, NE4 5PL, \\ UK and ${ }^{2}$ Human Nutrition Research Centre, Institute of Health and Society, Newcastle University, Newcastle upon Tyne, \\ $N E 24 H H, U K$
}

How dietary choices drive energy intake and affect adiposity is poorly understood. Traditional nutritional epidemiological methods of assessing the effects of a single, or a few, foods or nutrients on health, are now considered reductionist, since food is mostly consumed in meals, where each meal includes a variety of foods with differing combinations of nutrients that are likely to work cumulatively and synergistically. Dietary pattern analysis facilitates investigation of the effects of overall diet on health by attempting to characterise diets holistically. Cluster analysis classifies subjects into mutually exclusive homogeneous groups, based on individual differences in overall diet. This study characterised children's diets as dietary patterns using k-means cluster analysis and examined their relationships with adiposity.

Data were utilised from 468 children aged 6-8 years participating in the Gateshead Millennium Study; a longitudinal birth cohort study in North East England ${ }^{(1,2)}$. Dietary intake was measured by FAST ${ }^{(3)}$ and adiposity as Body Mass Index (BMI) and waist circumference (WC). To facilitate interpretation of clusters, dietary food items were aggregated into 41 food groups and expressed as standardised (z-scores) and non-standardised mean daily number of portions, energy intake and \% total energy intake. To find the most suitable number of clusters, k-means cluster analysis was run several times to identify dietary patterns using $2-8$ clusters. A minimum sample size was set, where $\geq 10 \%$ of subjects must be present in every dietary pattern identified by each run of cluster analysis. The resulting clusters were then examined for interpretable dietary patterns. Relationships between cluster membership and adiposity were investigated by ANOVA, adjusting for covariates.

Three clusters were identified using standardised \% total energy intake/food group, characterised as "healthy", "processed food" and "breakfast cereals" dietary patterns. BMI and WC were significantly different between "processed food" and "breakfast cereal" clusters. Adiposity of children within the "healthy" cluster did not significantly differ from those in the other clusters. Birth weight $(P<0.001)$, child age $(P<0.001)$, maternal BMI $(P<0.001)$ and physical activity $(P<0.05)$ were significant covariates.

\begin{tabular}{|c|c|c|c|c|c|c|}
\hline & \multicolumn{2}{|c|}{ Healthy cluster } & \multicolumn{2}{|c|}{ Processed food cluster } & \multicolumn{2}{|c|}{ Breakfast cereals cluster } \\
\hline & Mean & $\mathrm{CI}$ & Mean & $\mathrm{CI}$ & Mean & $\mathrm{CI}$ \\
\hline Child BMI & 16.62 & $(16.14-17.09)$ & $16.60 *$ & $(16.30-16.89)$ & $17.28 *$ & $(16.87-17.70)$ \\
\hline Child WC (cm) & 56.37 & $(55.21-57.53)$ & $56.16^{*}$ & $(55.43-56.88)$ & $58.02 *$ & $(57.01-59.03)$ \\
\hline
\end{tabular}

$* P<0.05$ by Bonferroni adjustment for multiple comparisons.

Perhaps counter intuitively, BMI and WC were lowest in the "processed food" cluster and highest in the "breakfast cereals" cluster. This finding might be related to parental misreporting of dietary intake, or parents recognising higher adiposity in their children and adopting healthier dietary patterns prior to data collection.

1. Parkinson KN, Pearce MS, Dale A et al. (2011) Int J Epidemiol. 40, 308-317.

2. Gateshead Millennium Study (2012) http://research.ncl.ac.uk/gms/ (accessed May 2012).

3. Adamson AJ, Griffiths JM, Carlin LE et al. (2003) Proc Nutr Soc. 62, 84A. 